\title{
Recommendations for Safely Performing Major Head and Neck Surgery During the COVID-19 Pandemic: Experience with Implementation of a Workflow
}

\author{
Subramania Iyer ${ }^{1}(\mathbb{1}) \cdot$ Sobha Subramaniam $^{2} \cdot$ Beena Ravikumar $^{3} \cdot$ Rajesh Pai $^{3}$. \\ Dipu Satyapalan ${ }^{4} \cdot$ Merlin Moni $^{4} \cdot$ Krishnakumar Thankappan $^{1} \cdot$ Pramod Subash $^{1}$
}

Received: 18 June 2020/ Accepted: 25 August 2020/Published online: 2 September 2020

(C) The Association of Oral and Maxillofacial Surgeons of India 2020

\begin{abstract}
COVID 19 pandemic has affected the delivery of surgical services as a part of management of head and neck cancers all over the world. Since it may affect the overall cure as well as quality of life of these patients, it is necessary to continue providing surgical treatment but with minimal additional health hazards to the patient or the health care worker. For this, a workflow was formulated in a university teaching hospital in India with large head and neck cancer workload and implemented during the period of national lockdown. 125 major head and neck cancer cases were operated during this period out of which 25 patients were of high-risk status. Emergency (10\%) and semi-emergency $(83 \%)$ cases predominated with few electives. The number of noncancer reconstructive and craniomaxillofacial cases operated was 81 , out of which $25 \%$ was of emergency in nature. When compared to the data of similar period in the previous year, $60 \%$ of the workload in the cancer-related cases could be offered surgical treatment, whereas the noncancer cases operated were only $25 \%$. The workflow may be useful for all surgical departments in safely performing procedures during this pandemic or similar situations in future with suitable refinements.
\end{abstract}

Subramania Iyer

subu.amrita@gmail.com

1 Department of Head and Neck, Plastic and CMF Surgery, Amrita Institute of Medical Sciences, Kochi, Kerala, India

2 Department of Pulmonology, Amrita Institute of Medical Sciences, Kochi, Kerala, India

3 Department of Hospital Administration, Amrita Institute of Medical Sciences, Kochi, Kerala, India

4 Department of Infection Control, Amrita Institute of Medical Sciences, Kochi, Kerala, India
Keywords COVID pandemic $\cdot$ Surgical guidelines $\cdot$ Head and neck surgery $\cdot$ Workflow

\section{Introduction}

COVID 19 pandemic, apart from its direct effect on health due to the viral infection, has affected the health status of many individuals by its impact on the health delivery system in most of the countries. The fear of spread of the disease to the health care workers and other patients has made medical institutions to suspend most of the medical and surgical services. Even provision of emergency services has been carried out with caution by many hospitals since treating a patient with COVID inadvertently may lead to mass quarantine of the staff and shutdown of the institution.

The practice of head and neck oncology has been influenced greatly by the COVID 19 pandemic. The virus load being greatest in the oropharynx and nasopharynx, head \&neck cancer management involves high aerosol-generating procedures (AGPs) with high risk of transmission to treating personnel. As per guidelines put forth by several societies, there is a general agreement in avoiding complicated surgeries necessitating prolonged ICU stay and modifying treatment regimens. The effect of the delay or denial of surgical care for head and neck cancer patients will affect overall cure rate and quality of life of patients [1]. Considering that surgical treatment cannot be deferred in majority of patients without detrimental effects, our unit which is a high-volume head neck oncology centre devised a workflow for practising safe surgery when the lockdown period started. This workflow looked into information available from the published literature [2-9], guidelines issued by various professional bodies institutions and governmental agencies published online. Our institution is situated in the state of Kerala where the first case of COVID 
was reported in India, in a person who travelled from Wuhan. Subsequently, because of efficient containment activities undertaken by the government the initial spike was controlled but following easing of travel restrictions another spike has occurred and is continuing at the time of writing this report. This paper reports the experience of undertaking surgical management of head and neck cancers from the time the national lockdown was declared till the end of the phase three of lockdown when return of natives from abroad and other states of the country started. Comparison of the workload with that during same time period in the previous year is also made to assess the impact of the pandemic.

For prioritising the delivery of surgical services, the surgical procedures were triaged based on their urgency to three categories. In order to define the safety measures to be undertaken, patients were then categorised to COVID high- or low-risk groups. Various types of quarantine and availability of preoperative for RT-PCR tests were considered in this triaging. The grades of precautions were prepared in which the types of the personal protection equipment (PPE) required, type of preoperative and postoperative ward care, and the transfer protocols were included. General precautionary measures for surgical and anaesthetic teams were also prescribed. The initial part of the paper will discuss the workflow, and the experience with the surgical practise will be reported in the latter part.

\section{Development and Structure of the Workflow}

\section{(a) Categorisation of surgical procedures}

The head and neck cancer-related surgery was categorised to three types based on the their urgency

\section{E1: Priority elective procedures}

Thyroidectomy and parotidectomy for benign conditions, benign but locally aggressive jaw tumours.

E2: Semi-emergency procedures that can wait 7-14 days

All head and neck cancer surgeries including malignancies of thyroid and parotid, diagnostic endoscopy of suspected malignancies.

E3: Emergency procedures to be done at the earliest

Acute airway distress requiring tracheostomy, reexploration for failing flaps, acute bleeding, abscess requiring drainage.

\section{(b) COVID risk status of patient}

This was categorised to COVID positive, high risk or low risk. A COVID-positive case was one with positive PCR tests. High and low risks were defined using existing public health criteria ("Appendix"). Any patient with two or more points was considered high risk.

\section{(c) Testing for COVID status}

Of the two types of tests available, RT-PCR detects presence of virus and other tests presence of antibodies. The latter is used only for community screening, and the RT-PCR-based tests assume importance in the evaluation, especially when the patient is asymptomatic carrier. While using tests in the preoperative setting, several issues need to be considered. These include limited availability of testing facilities, poor sensitivity of single or multiple tests which can vary with the timing in relationship to the viral load in the body and method of collection of samples. The guidelines by governmental agencies regarding the use of tests also need to be adhered to. If only one test was possible (T1), it was done $48 \mathrm{~h}$ prior to surgery, and if two tests were possible, $\mathrm{T} 1$ was done at day $0 / 1$ and $\mathrm{T} 2,48 \mathrm{~h}$ prior to surgery. In cases which had a prolonged hospital stay and reoperations, no further testing was advised if they stayed within the hospital and were asymptomatic. For reoperations done within 2-3 weeks after discharge, no further testing was suggested if they stayed in a low-risk area and were home-quarantined. Usually, the test report is available in $24 \mathrm{~h}$ when done in our facility, but nay take longer when done in a government facility.

\section{(d) Quarantine}

Quarantining is accepted as a good way to identify potentially infected patients by observing for development of symptoms or signs of COVID. It also helps to protect them from getting infected in the waiting period. The period of quarantine is debatable with 28 days as the safest. 14 days seems to be more accepted based on evidence that 99\% of patients will develop symptoms within 14 days. But in semi-emergency situations 7-14 days may have to be opted for. Quarantine could be home or institutional. In a preoperative setting, the institutional quarantine within the hospital may be detrimental. Hence, alternate facilities in hospital guest house were made available for this purpose. The patient had to stay always in the room, and food was delivered to them. They have to maintain social distancing, hand hygiene and wear mask. If the patient belonged to a low-risk zone, home quarantine was acceptable. During patient and family counselling, they were educated about the perils of operating if the patient is harbouring the virus. The method of quarantine, i.e. keeping them isolated at home with minimum contact with 
others and the family members taking strict precautions in avoiding contact with others, was explained. While formulating the grade of precautions, the quarantine status was designated as Q0-Nil Q1-7 days, Q2-14 days and Q328 days of quarantine.

\section{(e) Personal protection equipment}

PPE is the most important protective measure, and the levels were defined as

Level 1: Disposable apron, gloves and surgical face mask (visor/goggles if AGP is present).

Level 2: Disposable impermeable surgical gown of highquality N95 mask visor/goggles shoe cover, surgical gloves.

Level 3: Full body Coverall, shoe cover, N95 mask, goggles/visor, multiple layers of gloves.

\section{(f) Grading of surgical precautions:}

Grade 1 Admission in usual ward, normal transfer protocols with $\mathrm{PPE}_{\text {level1 }}$, surgery with $\mathrm{PPE}_{\text {level2 }}$.

Grade 2 Admission in usual ward, surgery with PPE level3, special zone ICU care with staff wearing $\mathrm{PPE}_{\text {level2 }}$ for patients on tracheostomy, ETT on $\mathrm{T}$ piece or ventilator, nasal pack or had surgical procedures on oral cavity and pharynx and larynx. These patients are shifted to normal care ward when these conditions cease to exist.

Grade 3 Admission in COVID ward. Surgery to be done in negative pressure area. $\mathrm{PPE}_{\text {level3 }}$ for procedure. Postoperative care in COVID ward with $\mathrm{PPE}_{\text {level3 }}$ till PCR test is negative. For patient transfer from/to the operation theatre, staff wears $\mathrm{PPE}_{\text {level2. }}$. The patient/bystander wears 3-layer surgical/N95 mask while being transferred into the OT. Transfer out of the OT through a dedicated corridor (decontaminated later on) or a covered trolley (specially prepared).

All patients except those belonging to the high-risk group with less than Q-2 quarantine, and those undergoing emergency surgery were admitted to the normal ward

\section{(g) Choice of the precautions based on the category of the patient}

Based on the type of surgery/COVID risk status, the patients were assigned appropriate grade of precautions. Quarantine status was most important tool for ensuring the virus-free status. The RT-PCR-based tests were added to the protocol when they became available in the institution

\section{E1: Priority elective procedures}

Low-risk group-Attain Q2 preferably Q3 status, when RT-PCR tests possible one test to be negative.

- $\mathrm{Q} 2 / 3 \pm \mathrm{T} 1$-Grade 1 precautions.

High-risk group-Attain Q2 preferably Q3 status, when RT-PCR tests possible at least $\mathrm{T} 1$ preferably $\mathrm{T} 2$ to be negative.

- $\mathrm{Q} 2 / 3+/ 1 \mathrm{~T} 1$ or $2-$ Grade 1 precautions.

- Q2/3 T0-Grade 2 precautions.

E2: Semi-emergency procedures that can wait 714 days

Low-risk group-Attain Q1 preferably Q2 status, when PCR tests possible T1 to be done.

- $\mathrm{Q} 1$ or $2( \pm) \mathrm{T} 1-$ Grade 1 precautions.

High-risk group-Attain Q1 preferably Q2 status, when PCR tests possible T1 preferably $\mathrm{T} 2$ to be done.

- $\mathrm{Q} 1 / \mathrm{Q} 2+\mathrm{T} 1 / \mathrm{T} 2$ - Grade 1 precautions.

- Q2-T0-Grade 2 precautions.

- Less than Q2 with T0-Grade 3 precautions.

\section{E3: Emergency procedures to be done ASAP}

Both low- and high-risk groups.

- Apply Grade 3 precautions.

When testing facility is available swab to be taken prior to surgery/postoperative period.

Figures 1 and 2 show the patient screening pathway and the flowchart to decide patient category and precautions to be selected

\section{(h) Additional precautions}

The following principles were observed by our surgical and anaesthesia teams during this period.

All cases to be done in the presence of senior surgical staff to reduce the operating time.

Adequate time between the cases to allow proper sanitisation of the theatre.

Number of personnel in theatre to be limited to essential minimum circulating nurse/technician to have instruments and consumables inside the OT beforehand avoiding unnecessary external movements. 
Fig. 1 Risk stratification of patients
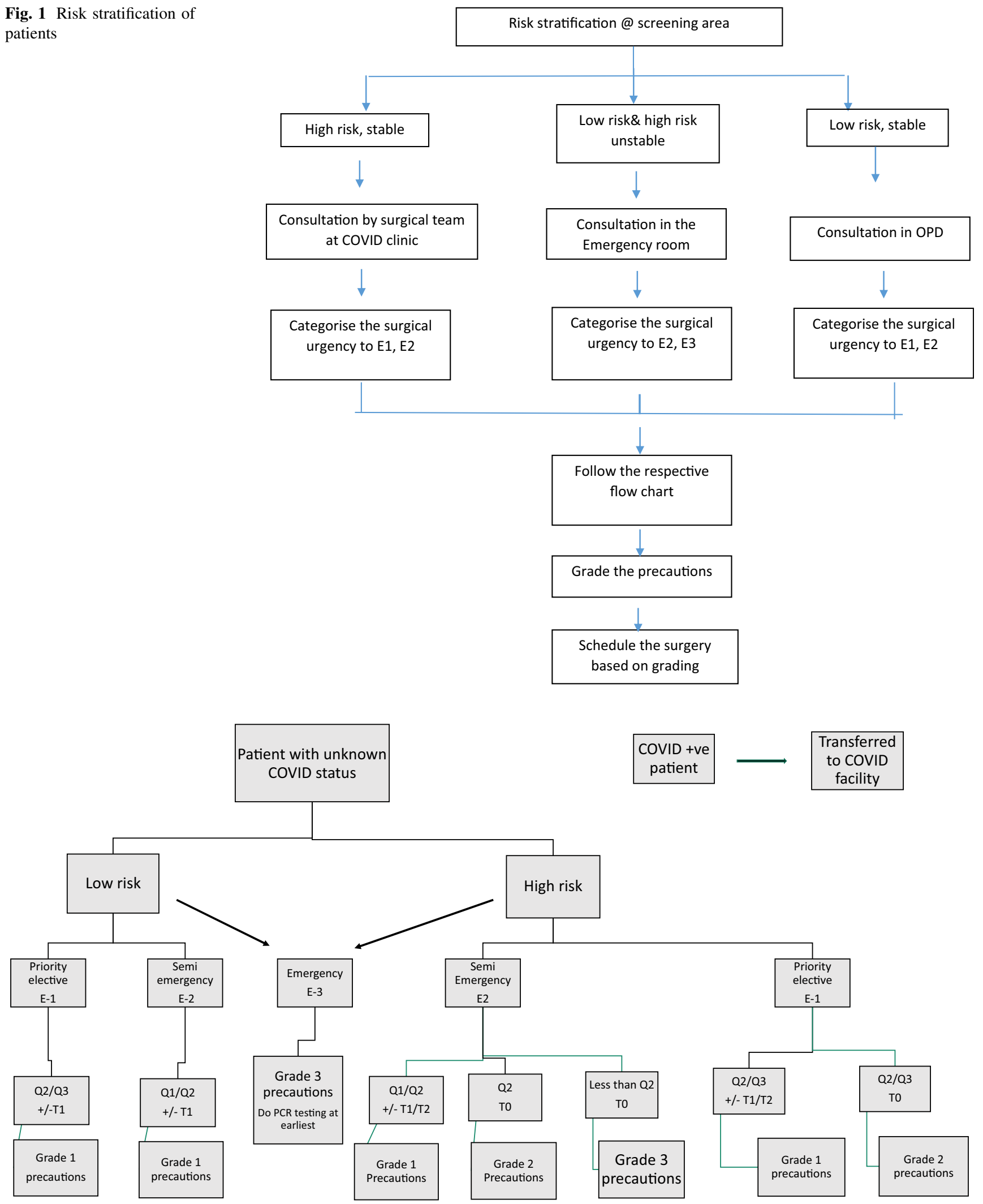

Fig. 2 Flow chart for deciding grade of precautions 
Cross-contamination vehicles like paper sheets, films, laptops not allowed inside OT

Theatre cleaned after procedure as per institutional infection control practices.

Anaesthesiology team took special care for aerosol generating procedures like intubation, extubation and endotracheal suction. Intubation to be performed by experienced anaesthesiologist to reduce the number of attempts. Extubation performed in the OR itself to minimize aerosol exposure to ICU staff.

A committee was formed to oversee surgical activities and help in triaging patients, choosing appropriate pathway and facilitating logistics.

\section{Details of Surgical Procedures Performed During this Period}

The hospital is a university teaching tertiary care hospital. COVID surveillance plan was initiated very early equipping with separate areas for taking care of the COVIDpositive patients. The period considered for the present study is from the time national lockdown was declared on March 25th to June 1st when considerable relaxation of the lockdown was made. This period had three distinct phases. Period A was Lockdown1 from March 25th to April 14th (17 days). During this time, there was no clear directive regarding the running of surgical services across the country except that all electives (E1) were to be stopped. The hospital workforce was reduced at this time as a precautionary measure. Only emergency (E3) and semiemergency (E2) cases were undertaken. During this time, a surgical services management group was formed consisting of senior surgeons, anaesthetists, members of infection control team and administration. The group developed these guidelines for surgical workflow described above. The infrastructure of the hospital also was augmented by creating negative pressure operation theatre suites as well as special areas for treating COVID high-risk/positive patients. The second phase was Lockdown 2 from April 14th to May 3rd (19 days). During this time, there was considerable reduction in the number of patients and the work consisted mainly of E2 and E3 cases. The workflow was enforced in the hospital with mainstay of the safety measure being triaging the patients and insisting on appropriate quarantining. During this period, effectiveness of the lockdown was good and the containment measures were strictly in place. The third phase was Lockdown 3-4 from May 4th to June 1st (26 days). This period reflects the easing of lockdown and travel restrictions from the other states and abroad. This was reflected in the steady increase in the number of the COVID cases reported in the state towards the end of this study period. In this period, the COVID PCR testing facility was available in the institution and could be used in some of the patients. The testing, however, was hampered by the extra cost, delay to get the results and stringer indications for testing prescribed by ICMR.

A total of 125 major head and neck cancer cases were operated during this period. The details of the numbers operated in these time periods as well as their triaging are given in Table 1. Majority of the patients were of low-risk status, but we operated on 25 patients $(20 \%)$ with high-risk status. Emergency cases consisted of $10 \%$, and majority $(83 \%)$ were of semi-emergency nature. The second period showed lesser cases, and number of electives increased in the third period.

The department offers reconstructive surgery for noncancer cases and all types of Craniomaxillofacial cases. Table 2 shows the number of these done in the department. Total of 81 cases were done out of which 19\% was from high-risk group. Relatively larger number of E3 cases were operated in this group (25\%).

A comparison was made in both the groups with the same time period in the previous year to find the impact on COVID pandemic on our surgical output. Figure 3 shows the comparison for head and neck cancers. Our output was about $60 \%$ of the workload operated during this period last year. The reduction is mainly in the E1 (elective) cases. During the current period, elective cases accounted only for $6 \%$, whereas in the corresponding time last year it was $35 \%$

Similar comparison for the noncancer cases is shown in Fig. 4. The surgical output was only $25 \%$ of previous

Table 1 Head and neck cancer cases operated during the three periods (lockdown 2020)

\begin{tabular}{lcccrr}
\hline & High risk & Low risk & E1 & E2 & E3 \\
\hline Period A (Lockdown 1) & 10 & 36 & 1 & 39 & 6 \\
Period B (Lockdown 2) & 6 & 18 & 0 & 23 & 1 \\
Period C (Lockdown 3) & 9 & 46 & 7 & 42 & 6 \\
Total & 25 & 100 & 8 & 104 & 13 \\
\hline
\end{tabular}

Table 2 Noncancer reconstructive, esthetic and craniomaxillofacial cases number of cases in categories, in three time periods (Lockdown 2020)

\begin{tabular}{lclrrr}
\hline & High risk & Low risk & E1 & E2 & E3 \\
\hline Period A (Lockdown 1) & 7 & 27 & 1 & 24 & 9 \\
Period B (Lockdown 2) & 4 & 10 & 3 & 9 & 2 \\
Period C (Lockdown 3/4) & 5 & 28 & 11 & 12 & 10 \\
Total & 16 & 65 & 15 & 45 & 21 \\
\hline
\end{tabular}


Fig. 3 Comparison with similar period in 2019- HN cancer patients
Number of Head and Neck Cancer Cases

Comparison between 2019 and the Lock-down

period

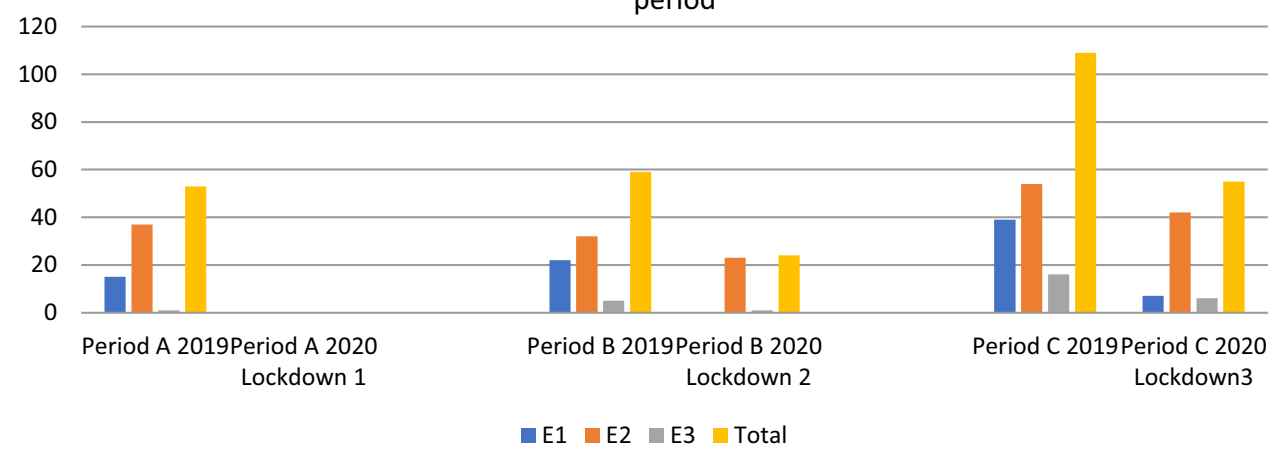

Non-cancer Reconstructive, Esthetic and Cranimaxillofacial cases

Comparison between 2019 and the Lock-down

period

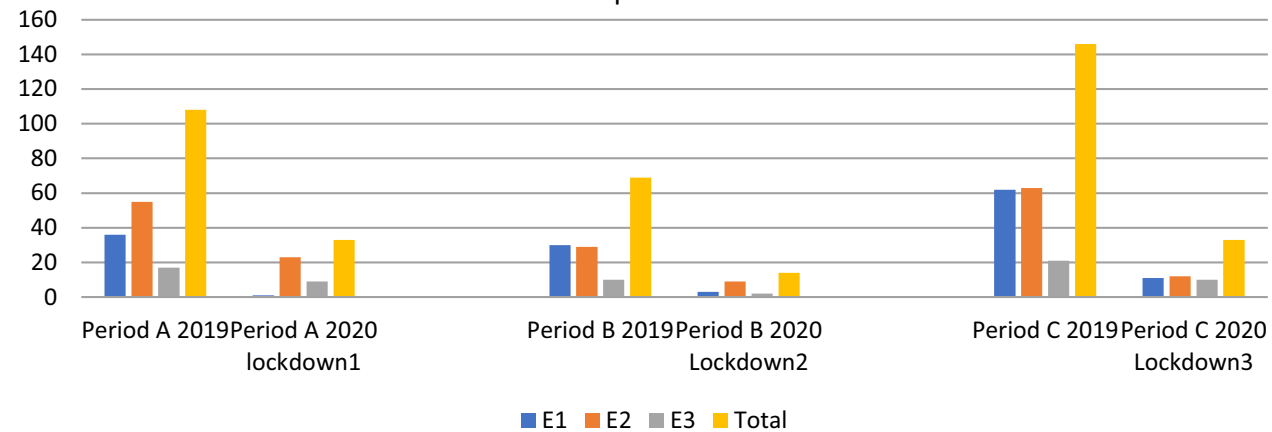

Fig. 5 Details of Quarentine and testing

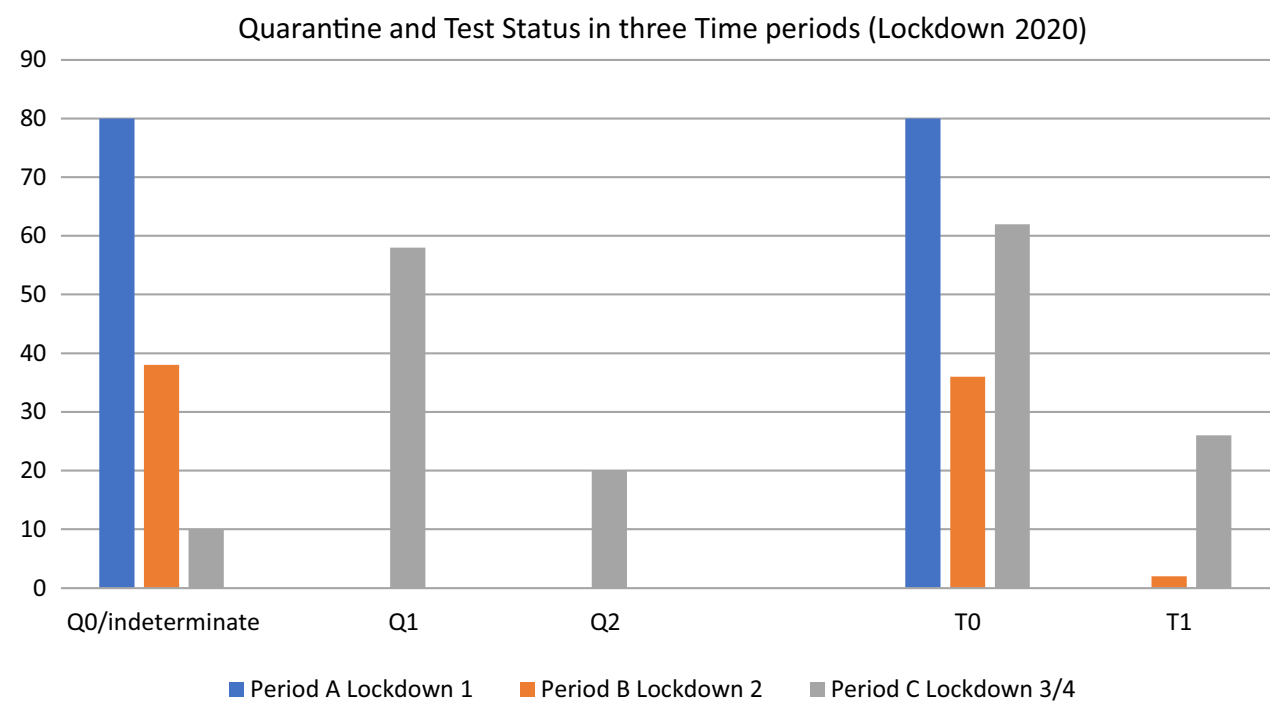

year's workload. The relative number of emergency cases was higher $25 \%$ versus $14 \%$.

Figure 5 shows details of quarantine and testing done during this period. During period A, quarantining concept was just starting as a preoperative precaution and was not recorded properly. But the use of quarantine increased in period. During period C, majority (90\%) of patients had 
undergone either Q1 or Q2. During this time, testing also became part of the precautionary measures.

During this period of the report, we could offer surgical care to 206 patients. Out of this, approximately 20\% belonged to the high-risk group. None of the patients operated developed any clinical evidence for COVID infection or tested positive. The number of cases operated during this period showed a definite dip from the same period last year. While comparing with the previous year cases, there was a gross reduction in the surgical output, but it was maintained more in head and neck cancer patients $(60 \%)$ than in noncancer patients $(25 \%)$. This may indicate our offering priority to these patients as well as willingness of the patients to seek surgical care during this time. The cases of head and neck cancers were offered standard of care including free flap reconstruction as before. It has to be emphasised that that this period doesn't really reflect a period of true community spread of the disease. When it occurs, more stringent testing and triaging might be necessary. However getting prepared in this way has equipped us better to offer normal standard of care treatment to the patients with head and neck diseases in the coming days where more number of COVID-positive or high-risk cases are expected. These guidelines may be useful for any high-volume center dealing with similar cases. Availability and more widespread use of PCR testing, testing of pooled samples, antibody screening tests of patients and health care workers may help in streamlining the triaging and use of precautions in a better way.

\section{Conclusions}

The present time has thrown great challenges in the health delivery system all over the world. This effect of COVID 19 has been most pronounced in the working of surgical services affecting proper delivery of care to head and neck cancer patients. Hence, it was necessary to restart the surgical services at the earliest. For this, a workflow based on available resources and knowledge of nature the viral pandemic was made. With such a workflow, we could ensure running the surgical services with more confidence and safety. When compared to a similar period, the workload equalled $60 \%$ in cases of head and neck cancer surgery, whereas there was a considerable drop of $75 \%$ in noncancer surgery. This workflow may become more relevant in the coming few months when the pandemic may persist. The workflow with refinements may be useful in safely performing surgery in similar situations in future also.

\section{Appendix}

Risk stratification criteria

(any person with 2 or more points go into the high-risk group)

- Symptoms complex suggestive of COVID like fever/ dry cough/breathlessness/throat discomfort/loose points) stools

- International/Out of state travel in the past 28 days (2

- Hailing from red zone or hotspot areas from other zones (gets updated on a timely basis)

- History of contact with suspected case of COVID 19 in quarantine

- Attending a gathering of more than 50 people in past (1 point) 14 days

- History of acute respiratory illness suggestive of $\quad$ (2 COVID 19

points)

- Critically ill patient with pneumonia during past 14 days positive case during past 14 days without appropriate PPE

- Travellers who visited a hospital where COVID $19 \quad$ (1 point) patients are treated

\section{References}

1. Day AT, Sher DJ, Lee RC, Truelson JM, Myers LL, Sumer BD et al (2020) Head and neck oncology during the COVID-19 pandemic: reconsidering traditional treatment paradigms in light of new surgical and other multilevel risks. Oral Oncol 105:104684

2. Lauer SA, Grantz KH, Bi Q, Jones FK, Zheng Q, Meredith HR et al (2020) The incubation period of coronavirus disease 2019 (COVID-19) from publicly reported confirmed cases: estimation and application. Ann Intern Med 172(9):577-582

3. Lei S, Jiang F, Su W, Chen C, Chen J, Mei W et al (2020) Clinical characteristics and outcomes of patients undergoing surgeries during the incubation period of COVID-19 infection. EClinicalMedicine 21:100331. https://doi.org/10.1016/j.eclinm.2020. 100331

4. Collaborative CO (2020) Mortality and pulmonary complications in patients undergoing surgery with perioperative SARS-CoV-2 infection: an international cohort study. Lancet. 396(10243): 27-38. https://doi.org/10.1016/S0140-6736(20)31182-X

5. Tran K, Cimon K, Severn M, Pessoa-Silva CL, Conly J (2012) Aerosol generating procedures and risk of transmission of acute respiratory infections to healthcare workers: a systematic review. PLoS ONE 7(4):e35797

6. Wong J, Goh QY, Tan Z, Lie SA, Tay YC, Ng SY et al (2020) Preparing for a COVID-19 pandemic: a review of operating room outbreak response measures in a large tertiary hospital in Singapore. Can J Anaesth 67(6):732-745 
7. Chen X, Liu Y, Gong Y, Guo X, Zuo M, Li J et al (2020) Perioperative management of patients infected with the novel coronavirus: recommendation from the joint task force of the Chinese Society of Anesthesiology and the Chinese Association of Anesthesiologists. Anesthesiology 132(6):1307-1316

8. Ti LK, Ang LS, Foong TW, Ng BSW (2020) What we do when a COVID-19 patient needs an operation: operating room preparation and guidance. Can J Anaesth 67(6):756-758
9. Brat GA, Hersey S, Chhabra K, Gupta A, Scott J (2020) Protecting surgical teams during the COVID-19 outbreak: a narrative review and clinical considerations. Ann Surg. https://doi.org/10.1097/ SLA.0000000000003926

Publisher's Note Springer Nature remains neutral with regard to jurisdictional claims in published maps and institutional affiliations. 